\title{
Generation of 224-nm radiation by stimulated Raman scattering of ArF excimer laser radiation in a mixture of $\mathrm{H}_{2}$ and $\mathrm{D}_{2}$
}

\author{
Genie G. M. Stoffels, Patrick Schmidt, Nico Dam, and J. J. ter Meulen
}

\begin{abstract}
Raman shifting of tunable ArF excimer laser radiation in a mixture of $\mathrm{H}_{2}$ and $\mathrm{D}_{2}$ produces tunable radiation in the 224-nm region as a result of Stokes shifting the frequency of the fundamental radiation (193 nm) once in both $\mathrm{H}_{2}$ and $\mathrm{D}_{2}$. At a total pressure of 25 bars, a $19 \% \mathrm{H}_{2}$ in $\mathrm{D}_{2}$ mixture is found to provide a maximum conversion efficiency $(2.5 \%)$ to the 224-nm range. Both fundamental and 224-nm radiation were used to record laser-induced fluorescence excitation spectra of nitric oxide produced in an oxyacetylene flame. From the excitation spectra, we determined the tuning range of the 224-nm radiation to be $270 \mathrm{~cm}^{-1}$ with a linewidth of $0.9 \mathrm{~cm}^{-1}$, which is similar to the fundamental laser radiation. We derived the exact Raman shift of the generated radiation by comparing both excitation spectra which was found to be $7142.3(5) \mathrm{cm}^{-1}$. (C) 1997 Optical Society of America
\end{abstract}

Key words: Stimulated Raman scattering, tunable excimer laser, mixture of $\mathrm{H}_{2}$ and $\mathrm{D}_{2}$.

\section{Introduction}

Nitric oxide (NO) is one of the principal pollutants formed in most combustion processes. Its presence can be demonstrated in situ by laser diagnostic techniques, ${ }^{1}$ among which laser-induced fluorescence (LIF), with use of tunable excimer or dye lasers for excitation, is probably the most widely used (see, e.g., Ref. 1). Within the tuning range of an excimer laser operated on $\mathrm{ArF}, \mathrm{NO}$ can be excited on the vibronic transition $D^{2} \Sigma^{+}\left(v^{\prime}=0\right) \leftarrow X^{2} \Pi\left(v^{\prime \prime}=1\right)$ around 193 $\mathrm{nm} .^{2} \quad$ At this transition only hot NO molecules, with a sufficient population of the $v^{\prime \prime}=1$ level, can be detected. Detection of cold NO molecules can proceed through the $A^{2} \Sigma^{+}\left(v^{\prime}=0\right) \leftarrow X^{2} \Pi\left(v^{\prime \prime}=0\right)$ band $(\lambda \approx 226 \mathrm{~nm})$, thus probing NO molecules in the vibrational ground state. Until now all experiments on the $A^{2} \Sigma^{+}\left(v^{\prime}=0\right) \leftarrow X^{2} \Pi\left(v^{\prime \prime}=0\right)$ transition have employed frequency-doubled dye laser systems yielding UV powers of a few millijoules at most. However, many applications require a higher power, particularly for two-dimensional imaging of NO.

\footnotetext{
The authors are with the Department of Molecular and Laser Physics, University of Nijmegen, Toernooiveld, NL-6525 ED Nijmegen, The Netherlands.

Received 23 January 1997; revised manuscript received 7 May 1997.

0003-6935/97/276797-05\$10.00/0

(C) 1997 Optical Society of America
}

Therefore it would be of interest to apply high-power tunable excimer lasers in combination with stimulated Raman scattering (SRS) to excite this transition. In this paper we describe experiments that show that it is possible to excite the $A^{2} \Sigma^{+}\left(v^{\prime}=0\right) \leftarrow$ $X^{2} \Pi\left(v^{\prime \prime}=0\right)$ transition by using narrow-band $\operatorname{ArF}$ excimer laser radiation that is Raman shifted in a mixture of $\mathrm{H}_{2}$ and $\mathrm{D}_{2}$.

The basic mechanisms of SRS of narrow-band radiation are well known. ${ }^{1,3,4,5}$ With use of a highintensity pump laser, the generated (first) Stokes line may acquire enough intensity to function as a pump for a higher-order (second) Stokes line. This process may repeat itself, so that a single pump laser of sufficient intensity may give rise to an entire progression of Stokes (and anti-Stokes) lines. By using a gas mixture as the Raman-shifting medium, we can also produce frequencies that are the result of subsequent interactions with the different constituents of the medium. So for mixed Raman media, lines with Raman shifts that are equal to the sum or difference of the vibrational frequencies of the components of the medium will occur. ${ }^{6}$ For example, in a mixture of $\mathrm{H}_{2}\left(\omega_{e}=4155 \mathrm{~cm}^{-1}\right)$ and $\mathrm{D}_{2}\left(\omega_{e}=2987 \mathrm{~cm}^{-1}\right),{ }^{7} \mathrm{a}$ line with a shift of $7142 \mathrm{~cm}^{-1}$ should also be produced. If we begin with a pump wavelength of 193 $\mathrm{nm}$, a wavelength of $224 \mathrm{~nm}$ would be reached. The occurrence of this line was demonstrated by Loree $e t$ $a l .{ }^{8}$ In this paper we describe experiments to determine the optimal $\mathrm{H}_{2}$ and $\mathrm{D}_{2}$ mixture for generating 


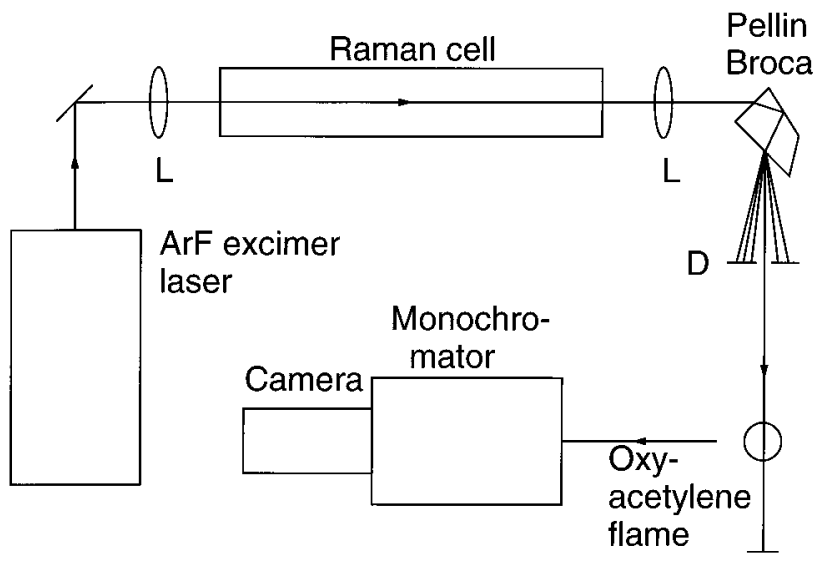

Fig. 1. Schematic view of the experimental setup: L, lens; D, diaphragm.

tunable radiation around $224 \mathrm{~nm}$, starting from an ArF excimer laser and its application to NO LIF on the $A^{2} \Sigma^{+}\left(v^{\prime}=0\right) \leftarrow X^{2} \Pi\left(v^{\prime \prime}=0\right)$ band.

We applied the LIF technique to measure NO excitation spectra using both the fundamental (193 nm) as well as the Raman-shifted (224-nm) radiation. We detected fluorescence from the $D^{2} \Sigma^{+}\left(v^{\prime}=0\right) \rightarrow X$ ${ }^{2} \Pi\left(v^{\prime \prime}=3\right)$ or the $A^{2} \Sigma^{+}\left(v^{\prime}=0\right) \rightarrow X^{2} \Pi\left(v^{\prime \prime}=2\right)$ transition around 208 and $248 \mathrm{~nm}$, respectively. We compared the 224-nm excitation spectrum with a spectrum measured by applying a dye laser system and with a simulated spectrum. From this comparison the influence of ambient oxygen on the 193-nm pump beam can be seen by a decrease of some of the strong lines in the spectrum measured with the Raman-shifted 224-nm radiation. We derived the net Raman shift by comparing both excitation spectra.

\section{Experiment}

A schematic view of the experimental setup is shown in Fig. 1. The laser used in the experiment is a pulsed tunable excimer laser consisting of two laser tubes in an oscillator-amplifier configuration (Compex 350T, Lambda Physik). The output of this laser, operated on $\mathrm{ArF}$, is tunable between 192.9 and 193.9 $\mathrm{nm}$ (tuning range of $270 \mathrm{~cm}^{-1}$ ) and has a linewidth of $0.9 \mathrm{~cm}^{-1}$. The laser can deliver a pulse energy as high as $350 \mathrm{~mJ}$, but in the current experiment typically $200 \mathrm{~mJ} /$ pulse is used. The shape of the beam is rectangular $(25 \mathrm{~mm} \times 3 \mathrm{~mm})$ and the pulse duration is $20 \mathrm{~ns}$. We achieved absolute frequency calibration by measuring the well-known $D^{2} \Sigma^{+}\left(v^{\prime}=0\right) \leftarrow X$ ${ }^{2} \Pi\left(v^{\prime \prime}=1\right)$ transition of NO (Ref. 9) in the same experiment.

To produce the Raman lines we focused the laser on a 185-cm-long, stainless-steel Raman cell filled with a mixture of $\mathrm{H}_{2}$ and $\mathrm{D}_{2}$. The cell was sealed hermetically by fused-quartz windows $(1.5 \mathrm{~cm}$ thick) and was designed to operate as high as 25 bars $(2.5 \mathrm{MPa})$. The emergent radiation, consisting of the fundamental laser frequency and all Raman-shifted frequencies generated in the cell, is collimated by a lens and the

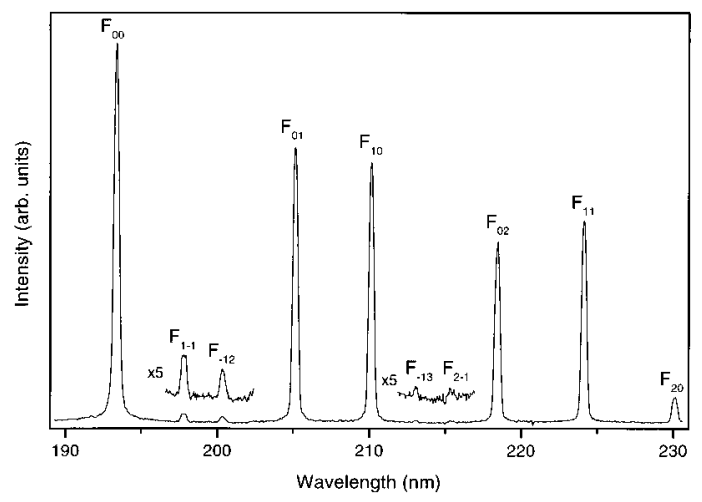

Fig. 2. Stimulated Raman scattering of ArF excimer laser radiation (at $193.4 \mathrm{~nm}$ ) in a mixture of $19 \% \mathrm{H}_{2}$ in $\mathrm{D}_{2}$ at a total pressure of 25 bars. The designation of the lines is explained in the text. The figure is not corrected for any wavelength-dependent detection efficiency.

individual components are separated by a PellinBroca prism. For LIF measurements the desired frequency is isolated by a diaphragm behind the prism and is focused by a cylindrical lens $(f=100$ $\mathrm{mm})$ in an oxyacetylene flame. The fluorescence is detected by a gated (20-ns), intensified CCD camera (ICCD-576G/RB-E, Princeton Instruments) placed behind a monochromator (Chromex 250i). The system is used as an optical multichannel analyzer to disperse the fluorescence in the flame. Alternatively, it can be used to identify the frequency components in the output of the Raman cell by looking at scattered light (the Pellin-Broca prism is, in this case, replaced by a mirror). The camera output is digitized by a controller (ST-138, Princeton Instruments) and sent to a computer for further processing.

\section{Results and Discussion}

When the laser beam is focused in the Raman cell that is filled with a mixture of $\mathrm{H}_{2}$ and $\mathrm{D}_{2}$, several Raman lines are generated. Shown in Fig. 2 is part of a spectrum obtained by Raman scattering of 193-nm radiation $\left(F_{00}\right)$ in a mixture of $19 \% \mathrm{H}_{2}$ in $\mathrm{D}_{2}$ at a total pressure of 25 bars. Lines are indicated by the symbol $F_{i j}$ in which $i$ and $j$ represent the component of the Stokes (positive) or anti-Stokes (negative) shift in $\mathrm{H}_{2}$ and $\mathrm{D}_{2}$, respectively. For example, $F_{1-1}$ is the line generated from the fundamental laser line by the combination of one Stokes shift in $\mathrm{H}_{2}$ and one anti-Stokes shift in $\mathrm{D}_{2}$. Clearly seen are the first and second Stokes lines of $\mathrm{H}_{2}\left(F_{10}, F_{20}\right)$ and $\mathrm{D}_{2}\left(F_{01}\right.$, $F_{02}$ ), which also appear if the cell is filled with pure $\mathrm{H}_{2}$ or $\mathrm{D}_{2}$, respectively. Also seen is an intense line at $224 \mathrm{~nm}\left(F_{11}\right)$, which is unique to the $\mathrm{H}_{2}$ and $\mathrm{D}_{2}$ mixture. This line is a combination of a first Stokes shift in $\mathrm{D}_{2}$ of the 210 -nm radiation $\left(F_{10}\right)$ and a Stokes shift in $\mathrm{H}_{2}$ of the 205-nm radiation $\left(F_{01}\right)$ (which, of course, have the same total Raman shift). Furthermore, some weak lines appear, all of which can be assigned to anti-Stokes shifts of a stronger Stokes line.

The data of Fig. 2 are produced under the mixture 


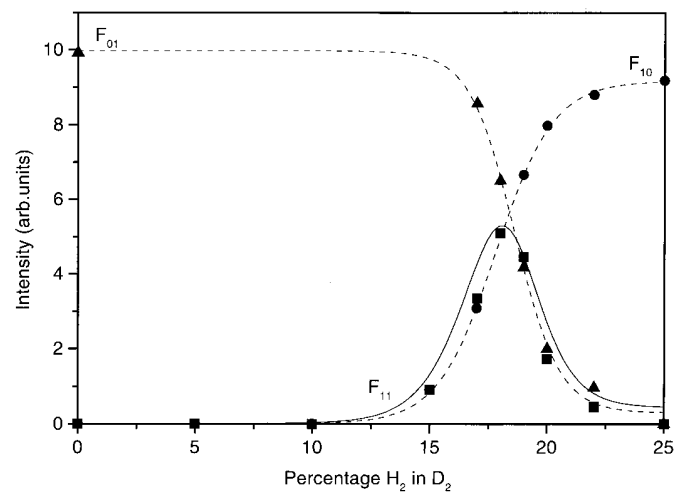

Fig. 3. Relative intensities of the first Stokes lines of $\mathrm{H}_{2}\left(\bullet, F_{10}\right)$, $\mathrm{D}_{2}\left(\boldsymbol{\Lambda}, F_{01}\right)$, and the 224-nm line $\left(\boldsymbol{\square}, F_{11}\right)$, as a function of the percentage of $\mathrm{H}_{2}$ in $\mathrm{D}_{2}$ (total pressure 25 bars).

conditions that were found to produce the most intense 224-nm $\left(F_{11}\right)$ line. We determined the optimal mixture by measuring the intensities of the first Stokes lines in $\mathrm{H}_{2}\left(F_{10}\right)$ and $\mathrm{D}_{2}\left(F_{01}\right)$ and the 224-nm $\left(F_{11}\right)$ line as a function of the mixing ratio for a constant total pressure of 25 bars. The result of this measurement is given in Fig. 3. The dashed curves for the $F_{10}$ and the $F_{01}$ lines are best fits (sigmoidal) through the measured data. Multiplication of these two fitted curves results (after rescaling) in the solid curve, which is seen to correspond well to the measured intensity of the $F_{11}$ line. This is not unexpected since the $F_{11}$ line is the combined result of Stokes shifting in both $\mathrm{H}_{2}$ and $\mathrm{D}_{2}$. From Fig. 3 it is clear that the 224-nm line is the most intense if the intensities of the $F_{01}$ and $F_{10}$ are equal. This is the case if approximately $19 \% \mathrm{H}_{2}$ is added to the $\mathrm{D}_{2}$ (at a total pressure of 25 bars). This corresponds to the ratio of the cross sections (and also the gain coefficients) of SRS in $\mathrm{H}_{2}$ and $\mathrm{D}_{2}$ : The Raman gain of $\mathrm{D}_{2}$ is approximately 5 times less than that of $\mathrm{H}_{2} \cdot{ }^{10} \mathrm{~A}$ conversion efficiency of $2.5 \%$ of the $224-\mathrm{nm}$ radiation $\left(F_{11}\right)$, resulting in $5 \mathrm{~mJ}$, is reached in this mixture. All further experiments are performed with the cell filled with $19 \% \mathrm{H}_{2}$ in $\mathrm{D}_{2}$ at a total pressure of 25 bars.

Figure 4 shows the intensity profiles of the fundamental 193-nm radiation $\left(F_{00}\right)$ in front of the Raman cell [Fig. 4(a)] and behind [Fig. 4(b)] the cell. There are some remarkable differences between the two plots. The intensity dips that are due to absorption of the 193-nm laser radiation by oxygen in the ambient air (Schumann-Runge bands ${ }^{11}$ ) is less pronounced in the profile measured behind the Raman cell. A possible explanation for this can be found in the fact that the intensity of the laser beam depends on absorption losses by atmospheric oxygen. Because SRS is a nonlinear process, small changes in initial laser intensity can have a pronounced effect on the Raman conversion efficiency and therefore through energy conservation on the pump depletion. It is to be expected, therefore, that a larger proportion of the laser intensity is Raman shifted the further the laser frequency is tuned away from oxygen absorption features. Consequently, the laser intensity de-

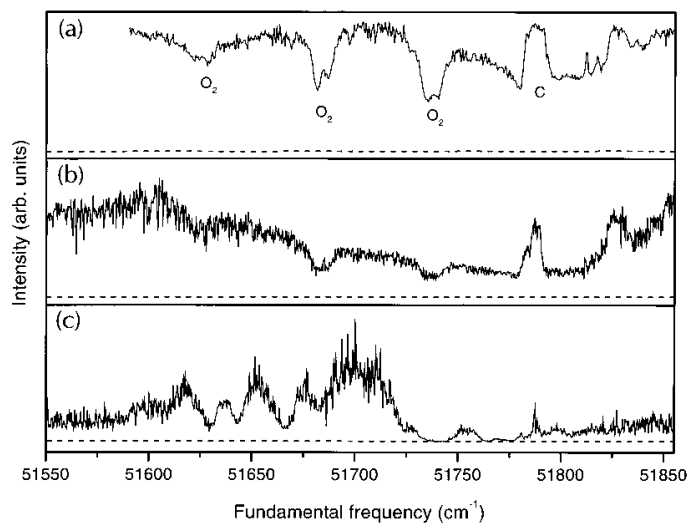

Fig. 4. Intensity profiles of (a) the $193-\mathrm{nm}$ radiation of the excimer laser, (b) the remaining $193-\mathrm{nm}$ radiation behind the cell $\left(F_{00}\right)$, and $(\mathrm{c})$ the generated $224-\mathrm{nm}$ radiation $\left(F_{11}\right)$ shifted by 7142.3 $\mathrm{cm}^{-1}$. Zero intensities are indicated by dashed lines.

pletion is strongest between the oxygen absorptions. This is confirmed by the intensity profile of the 224-nm radiation given in Fig. 4(c). It can be seen that, within the tuning range of the laser, the intensity of the 193-nm radiation drops and the intensity of the 224-nm radiation rises as a result of SRS in that range. When the pump beam is absorbed by oxygen, the intensity of the $224-\mathrm{nm}$ radiation drops, sometimes to zero as can be seen around $51,740 \mathrm{~cm}^{-1}$. The frequency range shown in Fig. 4 is somewhat larger than the tuning range of the excimer laser. Toward the tuning edges, the locking efficiency decreases and the laser becomes broadband. Part of this radiation is still Raman shifted, as can be seen from the noisy wings in Fig. 4(c). (Compare this with the zero intensity at the position of the strong $\mathrm{O}_{2}$ absorption at $51,740 \mathrm{~cm}^{-1}$.) It can be clearly seen in all three intensity profiles that fluorescence increases around $51,789 \mathrm{~cm}^{-1}$. This is due to an intracavity $\mathrm{C}$-atom absorption in the oscillator cavity of the ArF excimer pump laser at the $3^{1} P \leftarrow 2{ }^{1} D$ transition frequency, causing the laser to work broadband. ${ }^{12}$ (Note that this is in contrast to the $\mathrm{O}_{2}$ absorption that occurs in ambient air outside the laser cavity, thus decreasing the laser power and fluorescence intensity.) Figure 4 is recorded with an old laser filling in which apparently many $\mathrm{C}$ atoms were present, rendering the absorption feature as broad as $10 \mathrm{~cm}^{-1}$.

We determined the tuning range and linewidth of the 224-nm radiation $\left(F_{11}\right)$ by recording LIF spectra of NO in a flame. The $F_{11}$ component is used for excitation of NO at the $A^{2} \Sigma^{+}\left(v^{\prime}=0\right) \leftarrow X^{2} \Pi\left(v^{\prime \prime}=0\right)$ transition. The measured LIF spectrum is given in Fig. 5(a), together with the same part of this spectrum measured with a frequency-doubled dye laser working on Coumarin 47 [Fig. 5(b)]. Assignment of the spectra is achieved with the data from Reisel et al. ${ }^{13}$; a simulated spectrum $(T=1800 \mathrm{~K})$ is shown in Fig. 5(c). The oxygen holes in the gain curve of the $F_{11}$ line [Fig. 4(c)] are clearly reflected in the NO excitation spectrum of Fig. 5(a). There is good agreement of the $F_{11}$ spectrum with the dye laser 


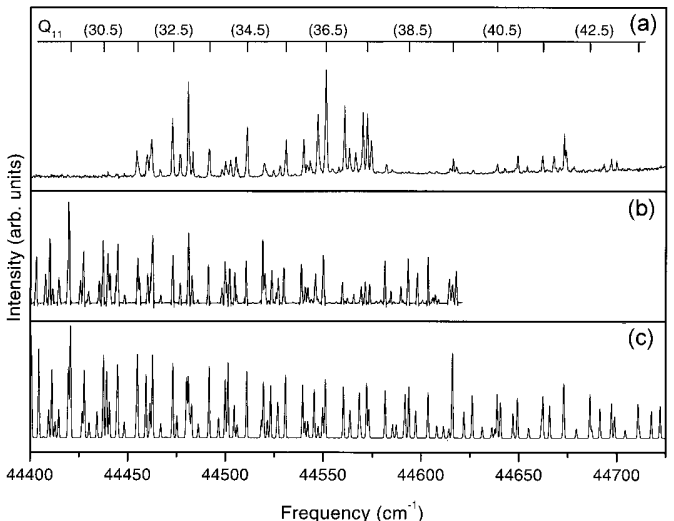

Fig. 5. Spectra of the $A^{2} \Sigma^{+}\left(v^{\prime}=0\right) \leftarrow X^{2} \Pi\left(v^{\prime \prime}=0\right)$ transition of NO (a) measured with the 224 -nm radiation $\left(F_{11}\right)$ generated in the Raman cell, (b) measured with a frequency-doubled dye laser, and (c) simulated with data from Reisel et al. ${ }^{13}$ Identification of the $Q_{11}$ branch is indicated.

spectrum and the simulated spectrum. The tuning range of the 224-nm radiation generated in the $\mathrm{Ra}-$ man cell is determined from the spectrum in Fig. 5(a) as $270 \mathrm{~cm}^{-1}$. This is equal to the tuning range of the pump laser. The linewidth of the 224-nm radiation is determined by a Gaussian fit through the $R_{11}(27.5)$ excitation line of the measured spectrum and is 0.9 $\mathrm{cm}^{-1}$, which is equal to the linewidth of the pump beam. Under the current experimental conditions this linewidth is predominantly attributable to the linewidth of the pump radiation. Therefore no measurable line broadening is caused by Raman shifting.

The remaining 193-nm radiation behind the Raman cell is used to record a LIF spectrum of NO on the $D^{2} \Sigma^{+}\left(v^{\prime}=0\right) \leftarrow X^{2} \Pi\left(v^{\prime \prime}=1\right)$ transition. This spectrum, given in Fig. 6, is well known and can be used for calibration of the pump laser frequency. ${ }^{9}$ After a comparison of the spectra from Figs. 6 and 5, the Raman shift was determined to be $7142.3(5) \mathrm{cm}^{-1}$ which is equal to the sum of the vibrational spacings of $\mathrm{H}_{2}$ and $\mathrm{D}_{2}$, as expected.

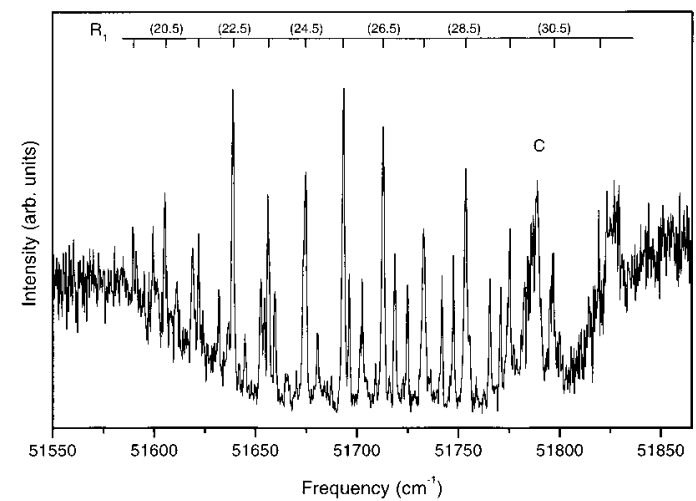

Fig. 6. Spectrum of the $D^{2} \Sigma^{+}\left(v^{\prime}=0\right) \leftarrow X^{2} \Pi\left(v^{\prime \prime}=1\right)$ transition of NO measured with the remaining 193-nm $\left(F_{00}\right)$ radiation behind the Raman cell. Identification of the strongest lines, $R_{11}$ branch, is indicated.

\section{Conclusion}

SRS of tunable, narrow-band 193-nm ArF excimer laser radiation in a $\mathrm{H}_{2}$ and $\mathrm{D}_{2}$ mixture is a useful technique to generate high-power, tunable, narrowband 224-nm radiation. The optimal condition for generating the most intense $224-\mathrm{nm}$ radiation (conversion efficiency of $2.5 \%$ ) is found to be a mixture of approximately $19 \% \mathrm{H}_{2}$ in $\mathrm{D}_{2}$ (at a total pressure of 25 bars). The effect of ambient oxygen on the pump beam is clearly reflected in the intensity profiles of the 224-nm radiation and the $193-\mathrm{nm}$ radiation in front of the Raman cell and behind the cell. The tuning range and linewidth of the generated 224-nm radiation are equal to those of the fundamental 193-nm radiation. By comparing NO excitation spectra recorded with either the fundamental or the Raman-shifted radiation, we found the net Raman shift to be $7142.3(5) \mathrm{cm}^{-1}$.

This scheme allows LIF detection of NO starting from both the $v^{\prime \prime}=0$ (on the $A \leftarrow X$ band at $224 \mathrm{~nm}$ ) and the $v^{\prime \prime}=1$ (on the $D \leftarrow X$ band at $193 \mathrm{~nm}$ ) with use of a single laser source. The power of the $224-\mathrm{nm}$ radiation that can be obtained by Raman shifting an ArF excimer laser exceeds the power delivered by frequency-doubled dye lasers in that region. Some obvious improvements of the experimental setup used here include antireflection-coated optics, thinner Raman cell windows, optimalization of the focus, and a purged beam path. If begun at $300 \mathrm{~mJ} /$ pulse, $>20$ $\mathrm{mJ} /$ pulse at $224 \mathrm{~nm}$ would be expected.

This research was financed by the Netherlands organization for applied scientific research and the Technology Foundation. Many enlightening discussions with W. L. Meerts are gratefully acknowledged.

\section{References}

1. A. C. Eckbreth, Laser Diagnostics for Combustion Temperatures and Species (Abacus, Cambridge, Mass., 1988).

2. Th. M. Brugman, R. Klein-Douwel, G. Huigen, E. van Walwijk, and J. J. ter Meulen, "Laser-induced-fluorescence imaging of NO in an $n$-heptan- and diesel-fuel-driven diesel engine," Appl. Phys. B. 57, 405-410 (1993).

3. R. W. Boyd, Nonlinear Optics (Academic, New York, 1992).

4. W. Demtröder, Laser Spectroscopy (Springer-Verlag, New York, 1996).

5. W. Kaiser and M. Maier, "Stimulated Rayleigh, Brillouin and Raman spectroscopy," Laser Handbook, Vol. 2, F. T. Arrechi and E. O. Schulz-Dubois, eds. (North-Holland, Amsterdam, 1972), pp. 1077-1150.

6. J. A. Duardo, L. J. Nugent, and F. M. Johnson, "Combination lines in stimulated Raman emission from gas mixtures," J. Chem. Phys. 46, 3585-3591 (1967).

7. D. C. Hanna, D. J. Pointer, and D. J. Pratt, "Stimulated Raman scattering of picosecond light pulses in hydrogen, deuterium, and methane," IEEE J. Quantum Electron. QE-22, 332-336 (1986).

8. T. R. Loree, R. C. Sze, D. L. Barker, and P. B. Scott, "New lines in the UV: SRS of excimer laser wavelengths," IEEE J. Quantum Electron. QE-15, 337-342 (1979).

9. M. Versluis, M. Ebben, M. Drabbels, and J. J. ter Meulen, "Frequency calibration in the ArF laser tuning range using laser-induced fluorescence of NO," Appl. Opt. 30, 5229-5234 (1991). 
10. S. K. Vartapetov and I. A. Veselovskii, "Stimulated Raman scattering of $\mathrm{KrF}$ laser radiation in deuterium," Quantum Electron. 25-1, 58-61 (1995).

11. M. P. Lee and R. K. Hanson, "Calculations of $\mathrm{O}_{2}$ absorption and fluorescence at elevated temperatures for a broadband argon-fluoride laser source at $193 \mathrm{~nm}$," J. Quant. Spectrosc. Radiat. Transfer 36-5, 425-440 (1986).
12. M. Versluis and G. Meyer, "Intra-cavity $\mathrm{C}$ atom absorption in the ArF laser tuning range," J. Chem. Phys. 96, 3350-3351 (1992).

13. J. R. Reisel, C. D. Carter, and N. M. Laurendeau, "Einstein coefficients for rotational lines of the $(0,0)$ band of the NO A ${ }^{2} \Sigma^{+} \leftarrow X^{2} \Pi$ system," J. Quant. Spectrosc. Radiat. Transfer 47-1, 43-54 (1992). 